\title{
Ex-Vivo Detection of Breast Cancer with a Bio-Impedance Sensor
}

\author{
Justina Ugwah ${ }^{5}$, Niall Savage', Walter Messina', Yineng Wang ${ }^{5}$, Edel Whelton ${ }^{5}$, Ann-Marie O'Donovan ${ }^{3}$, \\ Martin J.O'Sullivan'2, Brian D.O. Donnell ${ }^{2,4}$, Eric J. Moore ${ }^{1,5 *}$ \\ 'Life Sciences Interface, Tynadall National Institute, University College Cork, Ireland \\ ${ }^{2}$ Department of Surgery, Cork University Hospital, Cork \\ ${ }^{3}$ Department of Pathology, Cork university Hospital, Cork \\ ${ }^{4}$ Department of Anaesthesia, Cork University Hospital, Cork \\ ${ }^{5}$ School of Chemistry, University College Cork
}

\section{Article Info}

\section{Article Notes}

Received: February 16, 2021

Accepted: March 09, 2021

\section{*Correspondence:}

Dr. Eric J. Moore, Life Sciences Interface, Tynadall National Institute, University College Cork, Ireland;

E-mail: e.moore@ucc.ie

(c) 2021 Moore EJ. This article is distributed under the terms of the Creative Commons Attribution 4.0 International License.

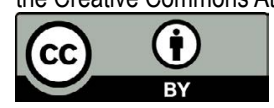

\section{Keywords:}

Breast Cancer

Bioimpedance

Ex-vivo characterization

\section{ABSTRACT}

Bioimpedance is the opposition to flow of an applied electrical current through biological tissues ${ }^{1}$. Our research group designed and fabricated bipolar micro-sensors on the tip of a silicone probe, capable of measuring biological tissue impedance. It is known that the bioimpedance of cultured cancer cells differs substantially from that of healthy cell lines. We hypothesised that the bioimpedance of cancer in surgically excised human tissue would be significantly different to surrounding healthy tissue. To test this hypothesis, we designed a study to evaluate the bioimpedance of healthy and diseased breast tissue in surgically excised breast specimens. This manuscript reports the outcome of this study.

\section{Introduction}

Cancer is a generic term for a large group of diseases characterized by the growth of abnormal cells beyond their usual boundaries that can invade adjoining parts of the body and/or spread to other organs ${ }^{2}$. According to WHO, as of 2016, cancer was the sixth most leading cause of death globally. Breast cancer is common and affects one in eight women ${ }^{3}$. It can arise from the milk ducts (ductal carcinoma $80 \%$ or from the breast stroma (lobular carcinoma $10 \%{ }^{4}$. Breast Cancer is genetically and histologically a heterogeneous disease that can be categorised into early or 'in-situ' or advanced or 'invasive' subtypes. Further classification is based on hormone receptor status, molecular biology and the degree of tumour spread ${ }^{5}$.

Early detection of breast cancer is central to improving patient outcome and lessening the risk of breast cancer associated death. Since 1990 in the USA, death directly attributable to breast cancer have dropped by $34 \%{ }^{3}$. Breast cancer screening programs improve survival from breast cancer ${ }^{6}$. Screening as seen in figure 1 , involves clinical physical examination, mammography and the histology of biopsy samples obtained from suspicious lesions found on mammogram ${ }^{7}$.

Bioimpedance is the opposition to flow of an applied electrical current through biological tissues. Our research group designed and fabricated bipolar micro-sensors on the tip of a silicone probe ${ }^{8}$, capable of measuring biological tissue impedance. It is known that the bioimpedance of cultured cancer cells differs substantially from that of healthy cell lines. We postulate that bioimpedance may play 
a role to improve the accuracy of biopsy samples obtained during breast cancer screening. To test this theory, it is important to first identify whether bioimpedance can differentiate between benign, malignant and normal breast tissue in patients with a confirmed diagnosis of breast disease.

To evaluate the bioimpedance of breast cancer in a clinical environment we designed a study to directly measure the bioimpedance of breast cancer in surgically excised breast cancer specimens.

\section{Methods}

\section{Ethical approval}

Ethical approval was granted by the Clinical Research Ethics Committee of Cork Teaching Hospitals to undertake clinical study. Study was performed at one center, Cork University Hospital, CUH. Study data were confidentially maintained and stored securely in accordance with the ethical approval application.

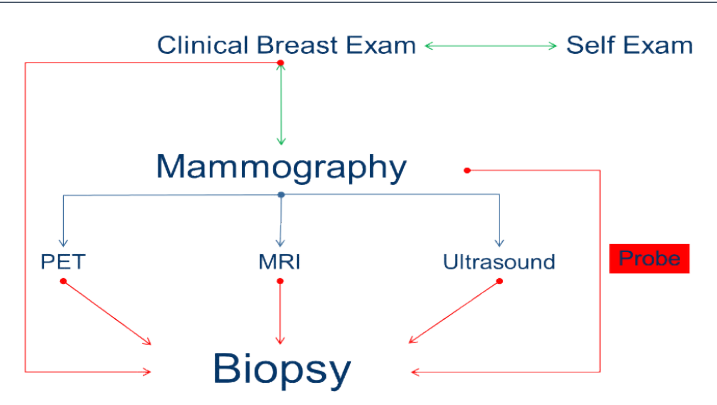

Figure 1: A schematic overview of the breast cancer detection pathway and the area in which the novel probe device fits within the pathway.

\section{Clinical study}

Patients scheduled for surgical excision of biopsy proven breast cancer with tumour size $>1.5 \mathrm{~cm}$ in diameter, irrespective of cancer subtype, were invited to participate in the study. Having provided informed written consent, patients were anaesthetized and underwent planned surgery. At surgery, the breast cancer was identified and excised together with a margin of healthy-looking tissue. After excision samples were placed in a dry specimen container without formalin. Specimens were taken to the histopathology laboratory within 15 minutes of excision, where a laboratory medical scientist (LMS) processed the sample as usual.

The LMS next marked (inked) and performed an initial tissue description in accordance with the SOP in the breast cut-up area of the histopathology laboratory. An incision was then made by the LMS to macroscopically identify both the tumour and normal breast tissue (Figure 2).

All impedance measurements were carried out with the Autolab PGSTAT204 with an FRA32M module (Metrohm U.K. Ltd., Cheshire, United Kingdom) with NOVA 1.1 software. Data-Therm IR thermometer (Guangxhou Jinxinbao Electronic Co. Ltd., Guangzhou, China. Harrick Plasma PDC-002 Extended Plasma Cleaner (Harrick Plasma, New York, U.S.A.).

The silicon probes were fabricated in Tyndall National Institute $^{8}$. The Autolab was set-up on the dissection bench and calibration protocol run each time by attaching the electrodes to the calibration box in the " $\mathrm{C}$ " configuration and the "Test FRA" method used to record 50 measurements across a range of frequencies. Once completed, the electrodes were then connected in a two-electrode system with the left and right electrode of the silicon probe

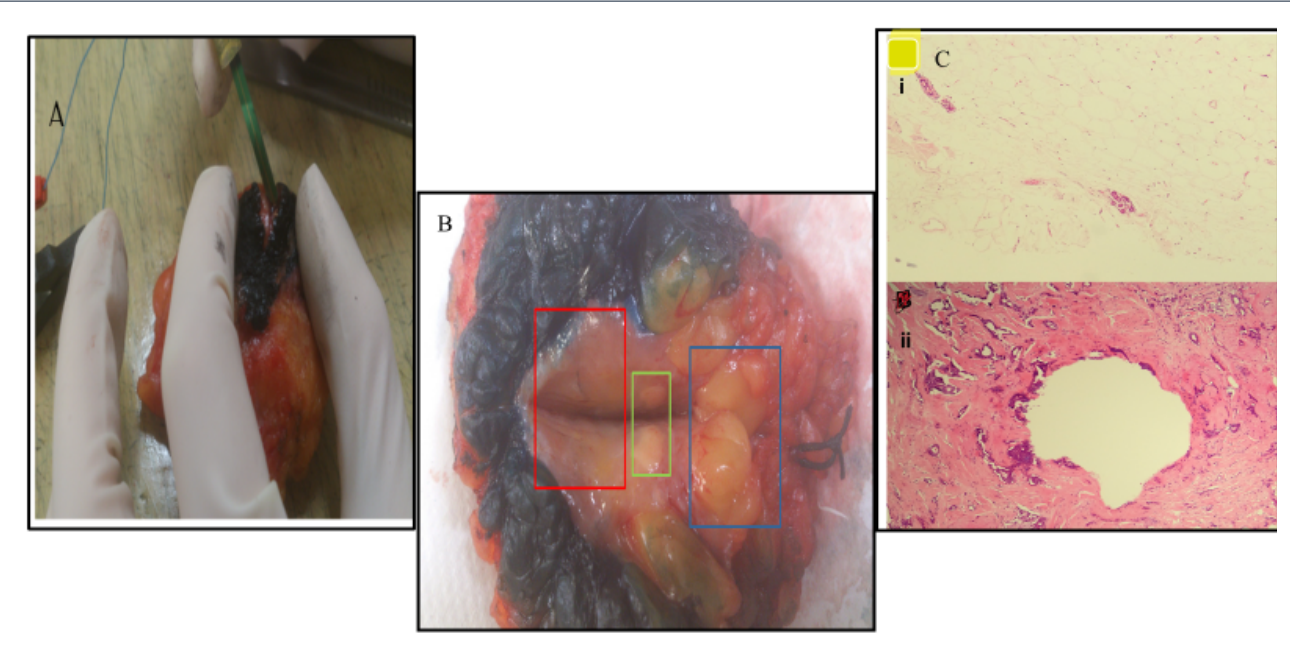

Figure 2A: The placement of a packaged probe in a cancerous lesion in a wide-local excision sample obtained from Study Participant 05. 2B: Shows the areas of interest in the wide-local excision sample of study participant 11 . The areas are the cancerous lesion (Red), fat necrosis (Green) and normal tissue (Blue). 2C: Shows the microscopic images obtained from the healthy tissue margin (A) and the cancerous lesion (B) of study participant 02 as confirmed by a consultant pathologist. 
attached to the sensing and working electrode respectively each time.

Frequency sweep measurements were performed on each of the patient samples, as no previous recordings had been performed on breast tissue using this probe. This data was then statistically analysed to determine an optimum frequency at which all future recordings could be performed. Triplicate recordings were obtained at each location across 30 frequencies from $100,000-1,000 \mathrm{~Hz}$ at $10 \mathrm{mV}$ amplitude. To determine the optimum current frequency for separation between normal and abnormal breast tissue impedance modulus, individual paired-samples t-test analyses were performed. Frequencies at either end of the spectrum were excluded, to avoid frequencies representative of resistive or capacitive elements.

The temperature of the tissue surface was measured with an infra-red thermometer prior to taking the first recording. The probes were cleaned twice using $18.2 \mathrm{M} \Omega$ nano-pure water and lint-free tissue between the healthy and cancerous recordings. The data was then exported to SPSS for analysis.

Once the tumour and healthy tissues had been identified by the LMS, the bioimpedance device was inserted into the incision site ensuring tissue to electrode surface contact by hand (figure $2 \mathrm{~A}$ ). For each specimen the area of cancer-free tissue was analysed before the cancerous lesion to prevent cross contamination. Both the points of electrode contact with cancer and cancer-free tissues were subsequently sampled histologically to cross reference with the bioimpedance data.

After final impedance recording, the sample was returned to the medical scientist to be covered with formalin and processed according to the histopathology protocol. A consultant pathologist microscopically analysed the samples and provided a pathologic description of the cells present in each. As part of the bioimpedance clinical study, the site of each probe recording was individually analysed to microscopically confirm the tissue type. The histological findings were aligned with the electrical impedance recordings, thereby facilitating a correlation of bioimpedance and histologic data. The pathologists report confirmed the tumour type and grade of each study participant's tissue samples.

\section{Results}

In accordance with the clinical trial protocol approved, electrical impedance recordings of ex vivo human tissue samples were conducted for 12 months in CUH. A total of 33 patients were recruited, however were 10 lost to followup as the surgical samples obtained were not suitable for electrical impedance recordings using the prototype silicone device. Excised breast tissues tested were obtained during mastectomy, central sector excision and wide-
Table 1. The Histological type of tumour with number of patients diagnosed and median age

\begin{tabular}{|l|c|c|}
\hline \multicolumn{1}{|c|}{ Tumour Type/Grade } & Age & Number of Patients \\
\hline Invasive Lobular Carcinoma Gr. 2 & 65 & 2 \\
\hline Invasive Ductal Carcinoma Gr. 2 & 60 & 7 \\
\hline Invasive Ductal Carcinoma Gr. 3 & 59 & 7 \\
\hline Mixed Invasive Ductal and Lobular Gr. 2 & 64 & 3 \\
\hline Invasive Mucinous Carcinoma Gr. 2 & 88 & 1 \\
\hline Metastatic Squamous Cell Carcinoma & 83 & 1 \\
\hline Fibroadenoma & 36 & 2 \\
\hline
\end{tabular}

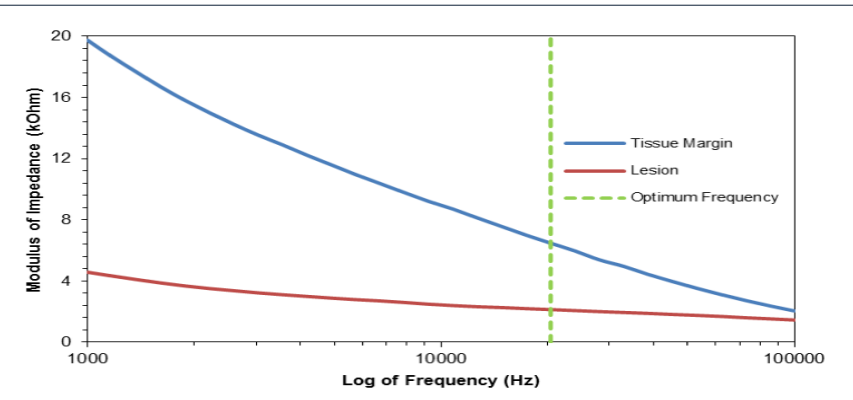

Figure 3: The Bode plot of the average impedance values for the healthy tissue margin and lesion, ${ }^{*} n=23$ (fibroadenoma included).

local excision surgeries. The histological diagnoses, age and tumour size of patients recruited to this study were evaluated as indicated in table 1.

A reliability analysis to identify discrepancies between the triplicate recordings obtained in each of the tissue locations was performed using a two-way random model to determine the intra-class correlation coefficient with a $95 \%$ confidence interval. The value of correlation was measured from 0 to 1 , with 1 being the highest degree of correlation between the data sets in this study. The InterRater Reliability, IRR analysis was also performed on the healthy, cancerous and benign data sets to determine the total cohort IRR values.

The data obtained from frequency sweep test indicated that the optimum frequency for recording of the combined tissue margin and lesions was 20,434 Hz. This showed that in all cases the silicon probe was able to differentiate between the healthy tissue and both benign and cancerous breast lesion at this frequency.

The mean difference at optimal frequency with 95\% confidence interval between the healthy tissue margin and lesion for all 23 patients analysed in this study was $4,340 \Omega$ with $\mathrm{p}<0.001$ (figure 3 ).

The statistical analysis of the 21 cancer patients samples in figure 4 with fibroadenomas excluded showed that, at the optimum frequency of $20,434 \mathrm{~Hz}$, the mean separation between the healthy breast margin and the cancerous lesions was $4,187 \Omega(p<0.001)$. This demonstrated that the prototype device was capable of differentiating between the two tissue types. 


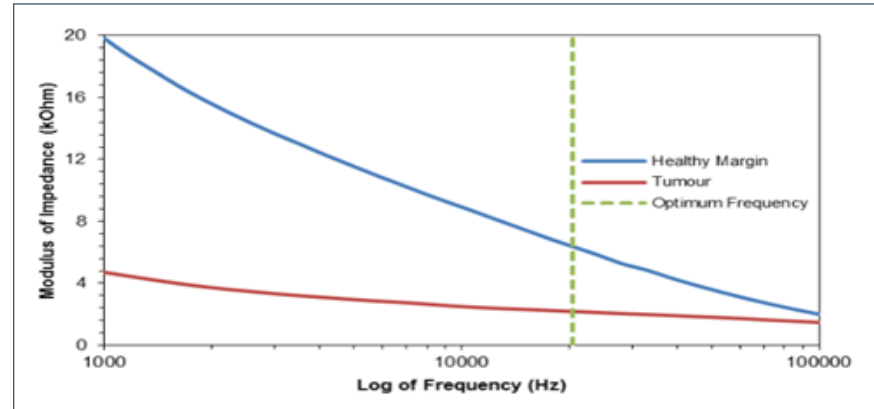

Figure 4: The Bode plot of the average impedance values for the tissue margin and cancer tumours. ${ }^{*} n=21$.

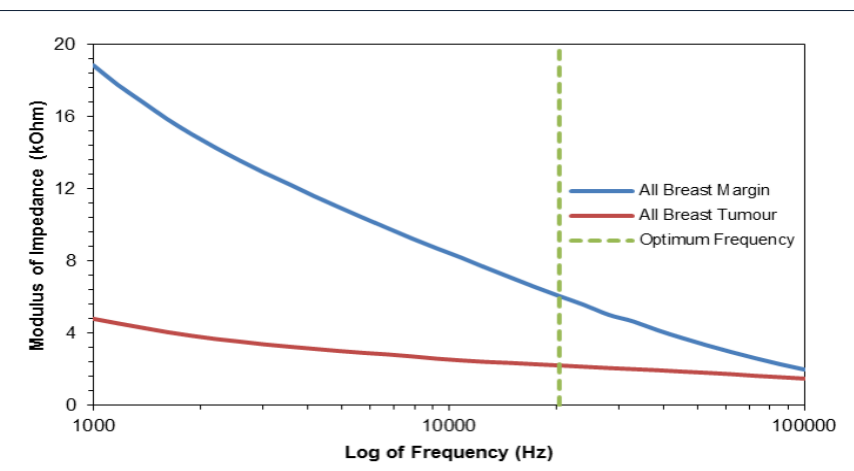

Figure 5: The average impedance values for the tissue margin and tumour for primary breast cancers. ${ }^{*} n=20$.

Figure 5 shows the paired-sample t-test showed that the mean separation between the primary breast cancer tumours; this excludes the metastatic squamous cell carcinoma, MSCC and the healthy tissue margins was $3,845 \Omega(p<0.001)$. From figure 5 we can see that there is evidence of separation.

Although the MSCC sample outlined in figure 6 was not a primary breast cancer it was included in the study to demonstrate the probes ability to differentiate between tumours that originated outside of the breast and the surrounding healthy breast tissue margin. The Bode plot of the recorded triplicate impedance values showed that a clear separation was present across all 30 data points measured. It was not possible to perform a paired-samples t-test as only one set of patient data was obtained for this cancer subtype. However, the mean values at the optimum frequency was still significant.

Two patients in the study with benign lesion, a fibroadenoma; tumour formed of mixed fibrous and glandular tissue, were analysed in figure 7 . At the optimum frequency they showed a statistically significant difference between the healthy tissue margin and the benign lesion $(5,947 \Omega ; \mathrm{p}=0.029)$.

\section{Discussion \& Conclusion}

This study demonstrated that a bipolar bioimpedance sensor mounted on a silicone probe can reliably measure

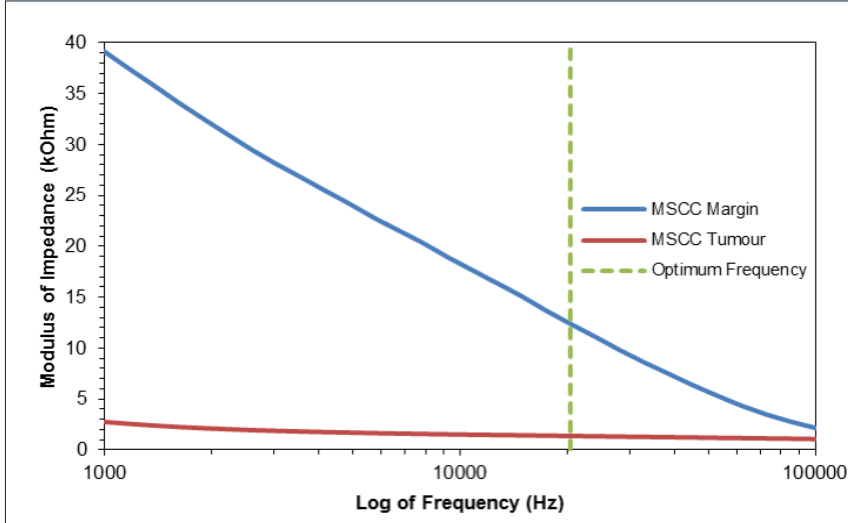

Figure 6: The Bode plot of the electrical impedance recordings taken from the metastatic squamous cell carcinoma (MSCC) specimen. $\mathrm{n}=1$.

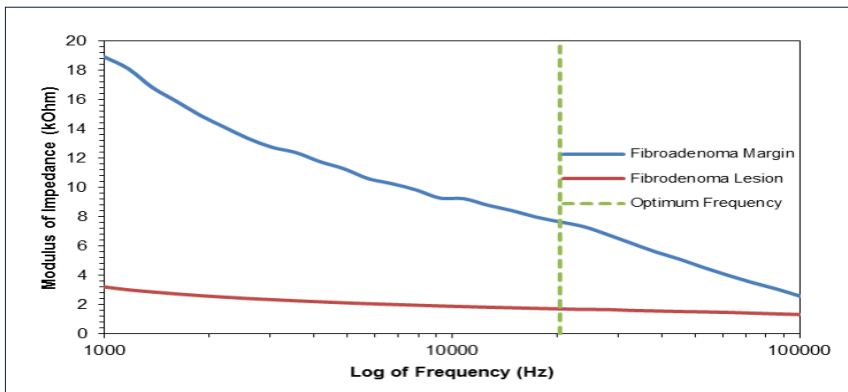

Figure 7: The Bode plot of the averaged electrical impedance results of the fibroadenoma patients consented to the clinical trial. ${ }^{*} n=2$.

the impedance of both healthy breast tissue and many types of cancer. The bioimpedance readings obtained suggest the utility of this technology in cancer diagnostics. Previous studies have also demonstrated that bioimpedance can be used to differentiate between healthy, cancerous and benign tissues ${ }^{9-11}$. Limitations to previously reported bioimpedance recording devices are largely due to electrode configuration and signal interference by tissues other than those of interest (e.g. surface electrodes and skin, sweat, hair etc.) In our study, a two gold microelectrodes patterned onto a passivated silicon substrate directly contacted the tissue of interest, thereby negating potential confounders.

The bioimpedance prototype device was capable of differentiating healthy breast tissue from breast lesions. In each of the tissue samples tested, separation in the bioimpedance signal between normal tissue and breast lesions increased as the frequency decreased. There appears to be a unique bioimpedance signal for each cancer subtype, although the sample size is too small to draw appropriate conclusions. The sample size is also too small to consider the sensitivity and specificity of the device as a diagnostic tool. 
Unfortunately, ten study participants were excluded from data collection in the laboratory. This was due to challenges in macroscopically identifying their tumour on initial tissue specimen processing. It is uncertain what, if any, the inclusion of data from these ten participants might have had on the overall study outcome.

The next iteration of this project is to integrate the sensors onto a needle, and validate by repeating the reported study in a large cohort of women undergoing breast cancer surgery. ultimately end application uses will be investigated through large multicenter studies. End applications for this technology may seek to enhance the accuracy of core needle biopsy.

\section{Data Availability Statement}

The datasets generated during and/or analysed during the current study are available from the corresponding author on reasonable request.

\section{Acknowledgements}

The authors acknowledge the assistance of the histopathology laboratory of Cork University Hospital, Cork, Ireland.

\section{Funding}

The study was organized as a joint project between Cork University Hospital and the Tyndall National Institute as part of a $\mathrm{PhD}$ research funded as part of the Clinical and Translational Research Scholarship Programme by Molecular Medicine Ireland.

\section{Conflict of Interest}

The authors declare that they have no known competing financial interests or personal relationships that could have appeared to influence the work reported in this paper.

\section{References}

1. Sarode GS, Sarode SC, Kulkarni M, Karmarkar S, Patil S. Role of bioimpedance in cancer detection: A brief review. Int J Dent Sci Res. Published online 2016. doi:10.1016/j.ijdsr.2015.11.003

2. World Health Organisation [Online]. https://www.who.int/newsroom/fact-sheets/detail/cancer

3. DeSantis CE, Lin CC, Mariotto $\mathrm{AB}$, et al. Cancer treatment and survivorship statistics, 2014. CA Cancer J Clin. Published online 2014. doi:10.3322/caac. 21235

4. Dossus L, Benusiglio PR. Lobular breast cancer: Incidence and genetic and non-genetic risk factors. Breast Cancer Res. Published online 2015. doi:10.1186/s13058-015-0546-7

5. Perry N, Broeders M, Wolf C de, Törnberg S, Holland R, Karsa L von. European Guidelines for Quality Assurance in Breast Cancer Screening and Diagnosis.; 2006. https://www.ccref.org/pro/pdf/reperes/ ND7306954ENC_002.pdf

6. Dean PB, Smith RA, Chen SL, et al. Early detection of breast cancer rectifies inequality of breast cancer outcomes. doi:10.1177/0969141320921210

7. Zhang Y-J, Wei L, Li J, Zheng Y-Q Li X-R. Status quo and development trend of breast biopsy technology. Gland Surg. Published online 2013. doi:10.3978/j.issn.2227-684X.2013.02.01

8. Ugwah JA, Savage N, Messina W, Donnell BDO, O’Sullivan MJ, Moore EJ. Fabrication of a Gold Dual-Microelectrode Patterned on a Silicone Substrate Probe for the Discrimination of Different Tissues. Electroanalysis. Published online 2020. doi:10.1002/elan.202060339

9. Morimoto T, Kinouchi Y, Iritani T, et al. Measurement of the electrical bio-impedance of breast tumors. Eur Surg Res. Published online 1990. doi:10.1159/000129087

10. Lee BR, Roberts WW, Smith DG, et al. Bioimpedance: Novel Use of a Minimally Invasive Technique for Cancer Localization in the Intact Prostate. Vol 39.; 1999.

11. Kinouchi Y, Iritani T, Morimoto T, Ohyama S. Fast in vivo measurements of local tissue impedances using needle electrodes. Med Biol Eng Comput. Published online 1997. doi:10.1007/BF02525528 